International Journal of Adhesion \& Adhesives 55 (2014) 89-94

http://dx.doi.org/10.1016/j.jjadhadh.2014.08.002

(C) 2014. This manuscript version is made available under the CC-BY-NC-ND 4.0 license

http://creativecommons.org/licenses/by-nc-nd/4.0/

\title{
Effect of annealing and silylation on the strength of melt-spun Ni-Mn-Ga fibres and their adhesion to epoxy
}

\author{
S. Glock, E. Spārninšs, Y. Leterrier and V. Michaud \\ Laboratoire de Technologie des Composites et Polymères (LTC), Ecole Polytechnique Fédérale de Lausanne \\ (EPFL), 1015 Lausanne, Switzerland
}

\begin{abstract}
Single crystals of ferromagnetic Ni-Mn-Ga shape memory alloys show magnetic-field and stress induced twinning, leading to shape memory. Adaptive composites can thus be produced by embedding single crystalline particles or bamboo-structured Ni-Mn-Ga fibres into a polymer matrix. To guarantee a durable performance of these composites, adhesion between reinforcement phase and matrix should be quantified and optimised. The influence of annealing and surface treatment with an aminosilane of melt-spun Ni-Mn-Ga fibres on their strength and adhesion to an epoxy matrix was investigated using single fibre tension and fragmentation tests. Annealing of melt-spun Ni-Mn-Ga fibres changed the surface from a "pimpled" to a smooth microstructure. This resulted in a reduced adhesion of the annealed fibres in comparison to the as-spun fibres embedded in an epoxy matrix. As-spun fibres exhibited an interfacial shear strength (IFSS) comparable to the shear strength of the epoxy matrix so that the silylation did not change the adhesion significantly. For the annealed fibres, the silane treatment improved the IFSS by $67 \%$. Furthermore, the silylation increased the fracture strength of the Ni-Mn-Ga fibres due to surface flaw healing or forming of a protective surface coating.
\end{abstract}

Keywords: A. Epoxides, B. Fibres, C. Silanisation, D. Interfaces, Magnetic Shape Memory Alloy, Fragmentation test

Corresponding author. Tel: +41216934923. E-mail address: veronique.michaud@epfl.ch. 


\section{Introduction}

Ferromagnetic shape memory alloys (FSMA), like Ni-Mn-Ga, show dissipative magnetic-field- and mechanical stress-induced twin boundary motion resulting in large reversible strains, up to $10 \%[1,2]$. Since these alloys are not thermally actuated, but instead show strain change in a magnetic field, the actuation frequency can reach up to $2 \mathrm{kHz}$ [3], making FSMAs interesting for applications requiring isothermal, high strain and high frequency actuation. In addition, the martensitic twinned structure at room temperature confers high damping to these alloys. In Ni-Mn-Ga polycrystals, however, twinning is hindered and the twinning stress is increased by the existence of grain boundaries. Ni-MnGa single crystals show optimal behaviour, but are expensive to produce and not available in complex shapes. A promising alternative to polycrystals and single crystals are composites with single crystalline $\mathrm{Ni}-\mathrm{Mn}-\mathrm{Ga}$ particles or $\mathrm{Ni}-\mathrm{Mn}-\mathrm{Ga}$ fibres with a bamboo structure (that is with grains as large as the fibre diameter) embedded in a polymer matrix [4].

In a composite, the adhesion between reinforcement phase and matrix depends on the morphology and chemistry of both surfaces in contact. To guarantee a good performance of the composite, in particular during actuation, a sufficient bonding between the reinforcement phase and the matrix is crucial. For fibre-reinforced composites, a set of experimental methods including pull-out test, microbond test or single fibre fragmentation test have been developed to determine the interfacial shear strength (IFSS) and are now routinely used [5]. The choice of a technique depends on the geometrical features of the fibre (diameter, available length), on the transparency of the matrix, and on the critical length of the fibre, which represents the embedded length below which the fibre strength 
cannot be reached and no fracture of the fibre can occur. For the pull-out and microbond tests, the embedded fibre length should remain below half of the critical length to prevent premature fibre failure. In contrast, for the fragmentation test the gauge length should be at least an order of magnitude longer than the critical fibre length to guarantee several fibre failures.

To improve the adhesion between reinforcement and matrix, several surface treatments of the reinforcement phase are possible [6]. These include the removal of weak boundary layers or contaminations, improvement of the wetting properties, chemical modification to link chemical groups at the surface, so that they further react with the matrix and finally, increasing of the surface roughness to improve the mechanical interlocking. A widely-used treatment to alter the surface chemistry of metal and metal oxide surfaces for adhesion with organic matrices are organofunctional silanes like $\gamma$ aminopropyltrimethoxysilane. These silanes contain an organic chain and alkoxy groups, which hydrolyse in water to form silanols. The silanol then condensates with the hydroxyl groups present on the metal surface to form covalent bonds. The inorganic metal surface is thus modified to an organic surface that can bond to the organic matrix $[7,8]$.

In this work the adhesion of as-spun and annealed melt-spun Ni-Mn-Ga fibres to an epoxy matrix was investigated. The two types of fibres were used without and with silane treatment. First, tensile tests were carried out to determine the strength of the individual fibres in the as-spun and annealed state and evaluate the potential influence of the silane treatment. Then, in order to investigate the effect of annealing and silane treatment on the interfacial strength of Ni-Mn-Ga fibres embedded in an epoxy matrix, single fibre fragmentation tests were performed and analysed. 


\section{Experimental}

The Ni-Mn-Ga fibres were produced by melt-spinning in the group of Prof. Zhang at the Harbin Institute of Technology, China following the procedure described in Ref. [9]. To obtain a bamboo-structure, about one half of the 100 fibres were annealed at $1000{ }^{\circ} \mathrm{C}$ for $3 \mathrm{~h}$ in a sealed, evacuated and argon backfilled quartz tube containing $\mathrm{Ti}$ (as oxygen getter) and $\mathrm{Mn}$ (to reduce Mn evaporation). Fig. 1 shows scanning electron micrographs of their surface and cross section, as-spun and after annealing. The fibres are irregular in shape, mostly half-circular in cross section and exhibit a fine dendritic surface after spinning but a smoother surface after annealing. The properties of the as-spun and annealed fibres, 30 to $100 \mu \mathrm{m}$ in width and up to $2.5 \mathrm{~cm}$ long, were determined and analysed in Ref.[10].
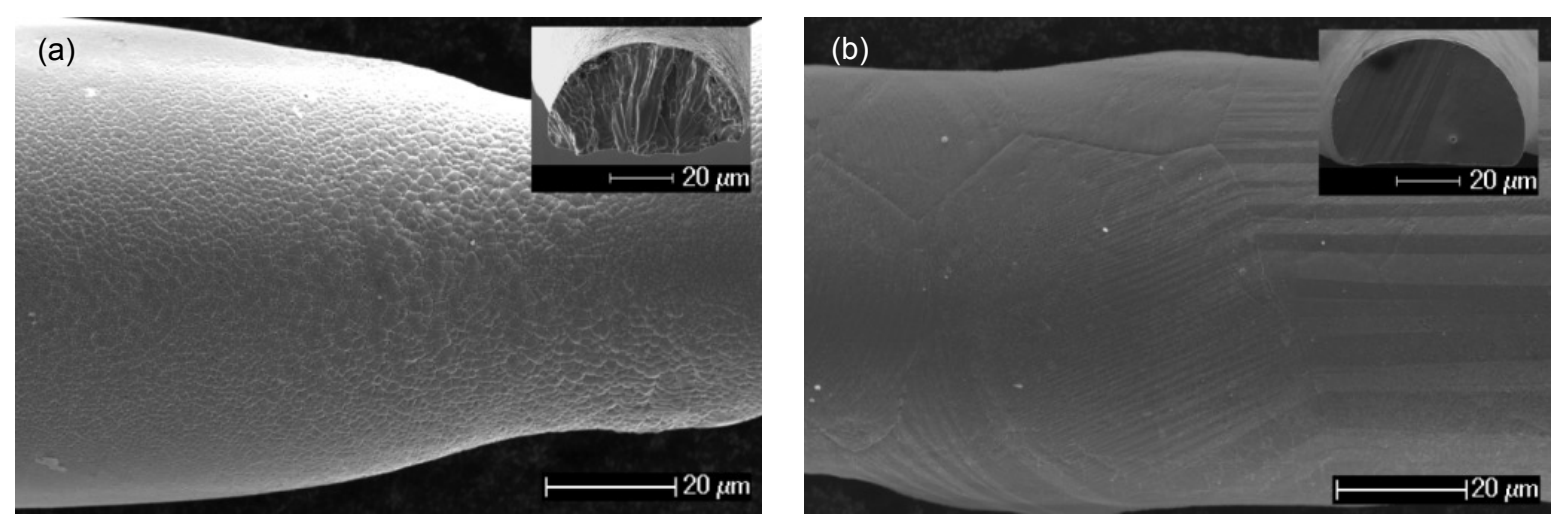

Fig. 1. Scanning electron micrographs of the surface and cross section of (a) an as-spun and (b) an annealed Ni-Mn-Ga fibre.

Silylation of the fibres was performed using a solution of 1 wt.\% $\gamma$ aminopropyltrimethoxysilane ( $\gamma$-APS, Silquest A-1100, Momentive, US), 1.5 wt.\% distilled water and $97.5 \mathrm{wt} . \%$ isopropanol mixed for $1 \mathrm{~h}$ at room temperature to hydrolyze the silane. Subsequently, the fibres were cleaned in acetone and isopropanol, dipped for $10 \mathrm{~min}$ in the 
silane solution and rinsed afterwards with isopropanol. Finally, the fibres were placed for $1 \mathrm{~h}$ in an oven at $120^{\circ} \mathrm{C}$ to cure the silane. The untreated fibres were cleaned in acetone before use.

The strength distribution, as well as the apparent tensile modulus and strain at failure of the as-spun and annealed, untreated and sylilated fibres, were determined in single fibre tensile tests up to failure. Tensile tests were performed on a tensile testing machine (UTS TestSystem Gmbh) equipped with a home-made $5 \mathrm{~N}$ load cell for gauge lengths of $1.5 \mathrm{~mm}$ and $3 \mathrm{~mm}$ for the silane treated and $1.5 \mathrm{~mm}$ to $13 \mathrm{~mm}$ for the untreated fibres. The gauge lengths as well as the number of samples were selected according to the availability of the fibre lengths. Each fibre was attached with cyanoacrylate-glue on the clamps and the test speed was $5 \mu \mathrm{m} / \mathrm{s}$. To evaluate the stress in the fibres, the cross section area at the location of failure was measured after each test.

In order to characterise the interfacial properties of all four fibre types, single fibre fragmentation tests were carried out. As matrix material, the epoxy system Araldite LY 3297/Aradur 3298 (with a mix ratio of 100:40 by weight) from Huntsman, USA was chosen. This standard epoxy system has a Young's-modulus of about $3 \mathrm{GPa}$ and a glass transition temperature at about $90^{\circ} \mathrm{C}[10]$. Dog-bone shaped specimens with one fibre centred in the specimen height and width were, due to the brittleness of the fibres, produced using a two step casting process. In the first step, liquid, degased epoxy was cast in a silicon mould and pre-cured at $80^{\circ} \mathrm{C}$ for $45 \mathrm{~min}$. The pre-cured epoxy specimen was then ground to obtain a flat surface. On the flat surface, a fibre was placed and fixed with a droplet of liquid epoxy at each end. After curing the droplets for $12 \mathrm{~h}$ at room temperature, liquid, 
degased epoxy was cast in a second step on the prepared specimen replaced in the silicon mould. To fully cure the epoxy, the specimen was placed in an oven at $80{ }^{\circ} \mathrm{C}$ for $9 \mathrm{~h}$. Finally, the cured specimen was ground and polished to obtain a thickness of $1 \mathrm{~mm}$ and a clear visibility of the fibre in the matrix. The final specimen dimensions are given in Fig. 2. Due to low availability of fibres with a sufficient length, the number of specimens was limited to 6 for the as-spun untreated fibres, 4 for the as-spun silane treated ones, 5 for the annealed untreated ones and 3 for the annealed silane treated ones. Preliminary investigations with dummy metallic fibres had shown that the epoxy/epoxy interface was not visible and did not influence the fragmentation test results.

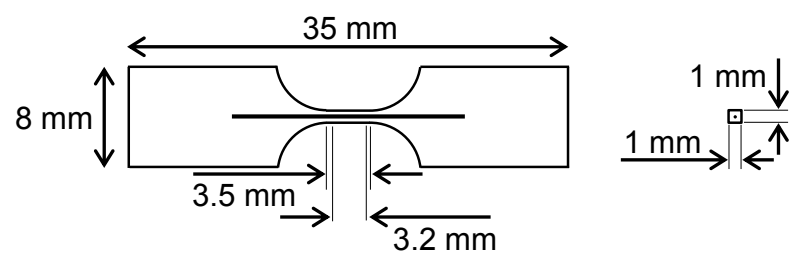

Fig. 2. Geometry of the fragmentation test sample. The gauge length was $3.2 \mathrm{~mm}$.

The fragmentation tests were conducted on a miniature tensile testing machine (TST350, Linkam, UK) equipped with a $200 \mathrm{~N}$ load cell. It was placed under a binocular lens between two perpendicularly oriented linear polarisers and the entire gauge length was observed in transmitted light. The displacement rate was set to $1.5 \mu \mathrm{m} / \mathrm{s}$ and a video of the fragmentation process was recorded during the test. In order to separate the fragments and thus to simplify counting of the fibre breaks, the samples were strained further than the ultimate elongation of the epoxy. The number of fragments was counted and the fibre width at the fracture location were measured under the microscope in unloaded specimens. For simplicity, in the following analysis of fragmentation tests, fibres were assumed to be 
circular in cross section, therefore the term "diameter" is used hereafter to describe the corresponding dimension.

\section{Results and discussion}

\subsection{Mechanical properties of the single fibres}

Fig. 3 presents the tensile behaviour of silane treated as-spun and annealed fibres. The as-spun fibres showed a brittle response, the annealed ones a serrated stress-strain behaviour with considerably lower stress. Furthermore, the annealed fibres broke along the grain boundaries. Thus, annealing of the fibres enabled twinning from the early stages of the test, resulting in significantly larger plastic strains. The untreated fibres behave in a similar way, as detailed in Ref. [10]. The silane treatment did not affect the general response of both fibre types to mechanical loading and seems not to hinder or block the stress induced twinning in the annealed fibres.

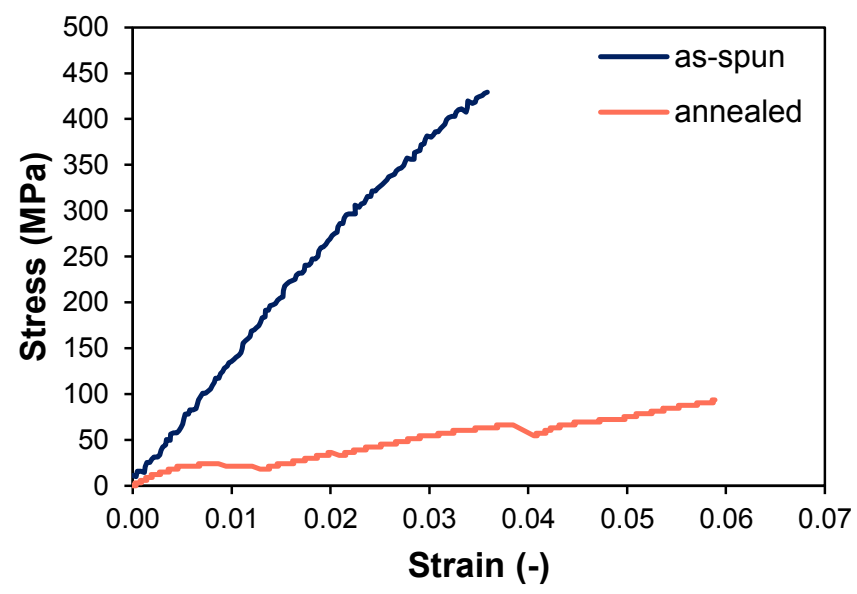

Fig. 3. Tensile behaviour of silane treated as-spun and annealed Ni-Mn-Ga fibres. 
Since no dependency of the strength on the fibre cross section was observed, the following two parameter Weibull distribution [11] was used to characterize the strength distribution of Ni-Mn-Ga fibres:

$$
P(\sigma)=1-\exp \left[-\frac{l}{l_{0}}\left(\frac{\sigma}{\beta}\right)^{\alpha}\right],
$$

where $\sigma$ is the fibre strength, $\alpha$ and $\beta$ are shape and scale parameters, respectively, of the Weibull distribution, $l$ designates the fibre gauge length and $l_{0}$ the reference length taken equal to $1 \mathrm{~mm}$ throughout the study. It follows from Eq. (1) that the average strength of the fibre is given by:

$$
\langle\sigma\rangle=\beta\left(\frac{l}{l_{0}}\right)^{-\frac{1}{\alpha}} \Gamma\left(1+\frac{1}{\alpha}\right)
$$

Since the number of tests performed at each gauge length was relatively small, it is not reasonable to show experimental distributions for each gauge length. Instead, the distribution of $\sigma\left(l / l_{0}\right)^{1 / \alpha}$ is presented for all fibre types in Fig. 4, where experimental probabilities were estimated as [12]:

$$
P=\frac{i-0.3}{m+0.4}
$$

where $i$ is the $i$-th number of ascendingly ordered strength data and $m$ is the sample size. Solid lines demonstrate the quality of approximation determined by the maximum likelihood method and can be interpreted as a fibre strength distribution at the reference length $l_{0}=1 \mathrm{~mm}$. An increase of the slope and a shift of the curve to higher stress levels due 
to the silane treatment are observed for untreated and silane treated fibres. The corresponding Weibull parameters for all four types of fibres are summarised in Table 1. The silane treatment did not affect the scale parameter of the Weibull distribution. It increased however the value of the shape parameter and thus reduced the scatter of fibre strength. Furthermore, as shown in Fig. 4 (c) and (d), the silane treatment slightly increased the average fibre strength for gauge lengths $l>l_{0}$.

Such an effect of the silane treatment has been reported previously for glass fibres [13-15]. Zinck at al. [13] attributed this increased strength to healing of pre-existing severe surface flaws with silane that penetrated and formed a three-dimensional network. As a result, the crack tip radius increased and stress concentrations were reduced. Due to a minimum hydrodynamic radius of aggregates of hydrolysed silane, the healing effect is limited to surface flaws that are large enough to allow sufficient interactions between flaws and aggregates. Another approach to explain the increase of the fibre strength due to a silane treatment was proposed by Yang et al. [15]. These authors assumed that silane forms a protective surface coating, which weakens further surface flaws caused by fibre manipulation and fibre/fibre interaction. The melt-spun Ni-Mn-Ga fibres tested in this work show a brittle failure in both, the as-spun and annealed states. Furthermore, the fibre manipulation before and after the silane treatment might have induced surface flaws. Thus, both healing of surface flaws and forming of a protective surface coating could be responsible for the increased strength of the silane treated Ni-Mn-Ga fibres.

The heat treatment of the fibres and resulting bamboo microstructure facilitate fracture along the grain boundaries. This leads to a scale parameter and average fibre 
strength that are about 6 times smaller than that of the as-spun fibres, Table 1 and Fig. 4 (c, d).
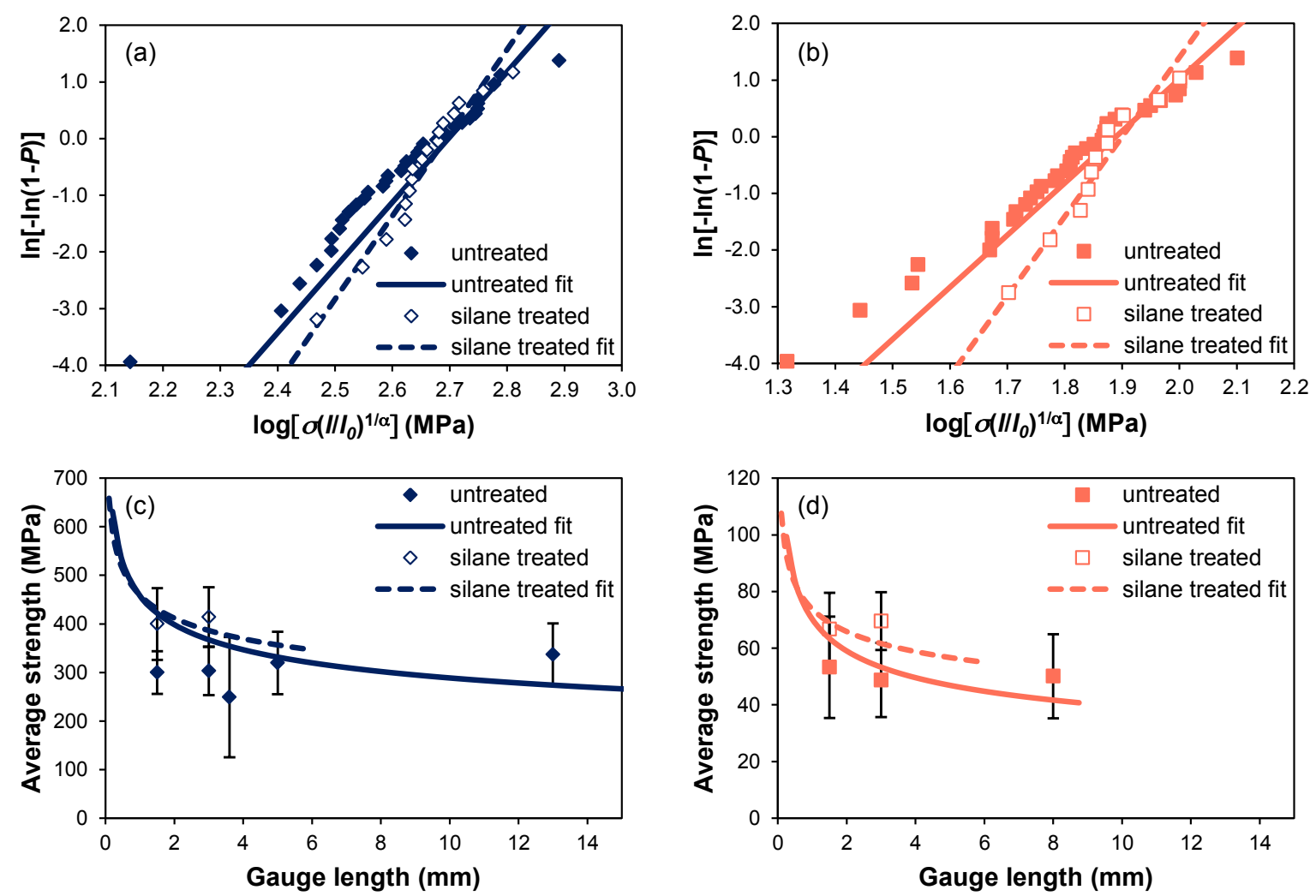

Fig. 4. Strength distribution of as-spun (a) and annealed (b) untreated and silane treated melt-spun $\mathrm{Ni}-\mathrm{Mn}-\mathrm{Ga}$ fibres for all gauge lengths; average strength of the as-spun (c) and annealed (d) fibres as a function of gauge length. The lines represent the Weibull distribution according to Eq. (1) in (a, b) and Eq. (2) in (c, d). 
Table 1

Properties of Ni-Mn-Ga fibres obtained from single fibre tension (SFT) and single fibre fragmentation (SFF) tests.

\begin{tabular}{lllllll}
\hline \multirow{2}{*}{ fibre type } & \multicolumn{2}{l}{ Shape parameter } & $\begin{array}{l}\text { Scale } \\
\text { (MPa) }\end{array}$ & parameter & $\begin{array}{l}\text { Modulus } \\
\text { (GPa) }\end{array}$ & $\begin{array}{l}\text { Strain at } \\
\text { failure (\%) }\end{array}$ \\
\cline { 2 - 5 } & SFT & SFF & SFT & SFF & & \\
\hline $\begin{array}{l}\text { as-spun } \\
\begin{array}{l}\text { as-spun } \\
\text { silane treated } \\
\text { annealed }\end{array}\end{array}$ & 5.0 & 5.1 & 498 & 464 & $18.6 \pm 7.8$ & $3.0 \pm 1.5$ \\
$\begin{array}{l}\text { annealed } \\
\text { silane treated }\end{array}$ & 6.4 & 5.0 & 493 & 424 & $18.7 \pm 4.8$ & $2.9 \pm 0.6$ \\
\hline
\end{tabular}

Table 1 summarises as well the average modulus and strain at failure for all four fibre types. The modulus, in particular, was estimated from the slope of the linear region of the stress-strain curve in the strain range from $0.05 \%$ to $0.25 \%$. It corresponds to an apparent (lowered) modulus in the case of the annealed fibres due to early anelastic plastic strain caused by twinning [10]. The scatter in the results, however, is too high to reveal any potential effect of the surface treatment on the modulus of the fibres.

\subsection{Fragmentation tests}

Fig. 5 presents a fragmentation test sample of an as-spun fibre in transmitted, polarized light. Six photo-elastic crosses within the gauge length along the Ni-Mn-Ga fibre, rotated by $45^{\circ}$ to the fibre axis are clearly visible. The corresponding stress concentrations in the matrix mark the positions of the fibre fractures. 


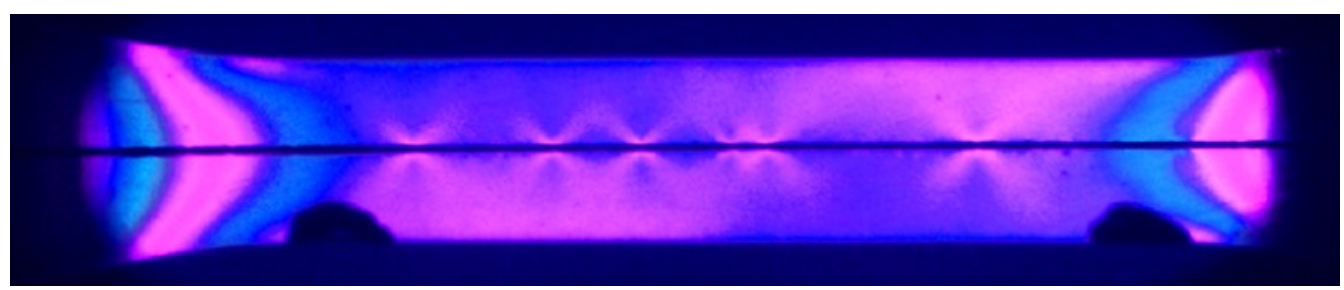

Fig. 5. Fragmentation test sample during the test in transmitted, polarized light. The six photoelastic crosses caused by stress concentrations mark the positions of the fibre fractures. (Note that two fractures in the middle of the sample are very close to each other.)

From the analysis of the videos recorded during loading of single fibre composites, classical fragmentation diagrams were constructed. Since the thermal expansion coefficients of the constituents were dissimilar, corrected fibre strains $\varepsilon_{f}=\varepsilon+\varepsilon_{r}$ ( $\varepsilon$ : strain obtained from the video, $\varepsilon_{r}$ : thermal residual strain) were used. The thermal residual strains were, due to a negligible fibre volume fraction in the specimen, estimated using following simplified expression:

$$
\varepsilon_{r}=\left(\alpha_{m}-\alpha_{f}\right) \Delta T
$$

where $\alpha_{m}$ and $\alpha_{f}$ are the matrix and fibre thermal expansion coefficients, respectively, and $\Delta T$ is temperature difference between curing $\left(80{ }^{\circ} \mathrm{C}\right)$ and testing temperature $\left(23^{\circ} \mathrm{C}\right)$. Using material properties of $\alpha_{m}=50^{*} 10^{-6} \mathrm{~K}^{-1}$ and $\alpha_{f}=15^{*} 10^{-6} \mathrm{~K}^{-1}[16,17]$, the residual strains in the fibre were evaluated as $-0.2 \%$. Fig. 6 shows a typical loading curve of one sample (a) and the fragmentation diagram for as-spun fibres (b). Failure onset was detected at about 1.5 to $2 \%$ strain. Additional failures occurred with increasing strain and proceeded beyond yielding of the matrix. For the annealed fibre specimens, fragmentation diagrams could not be obtained since the strength of the annealed Ni-Mn-Ga was too low to cause a stress 
concentration in the matrix that could be observed in the video. Thus, full fragmentation data were available and analysed only for as-spun fibres.
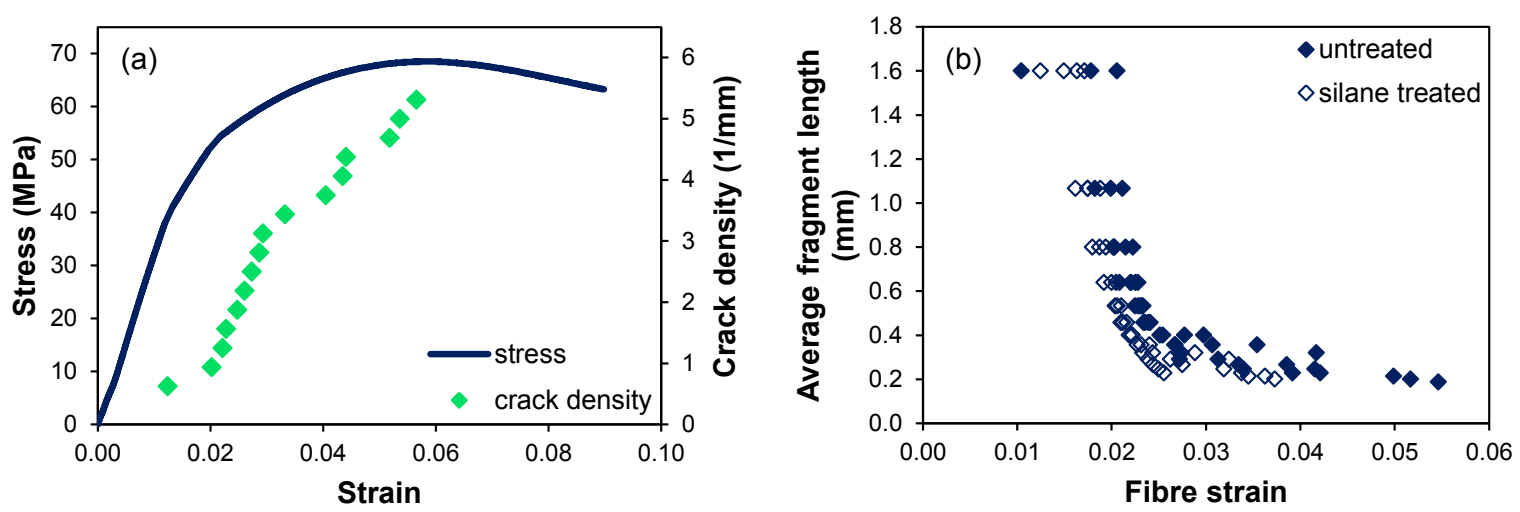

Fig. 6. Stress-strain curve of one fragmentation test sample of an untreated as-spun fibre embedded in an epoxy matrix (a) and average fragment length as a function of fibre strain for all as-spun single fibre composites (b).

Fig. 7 shows unloaded fractured fibres embedded in epoxy after the fragmentation test. In the case of the annealed fibre, fracture occurred only along the grain boundaries. Furthermore, debonding seems to have taken place around the fractures of the as-spun and annealed fibres during the subsequent loading after the onset of multiple resin necking at the locations of fibre fractures. 

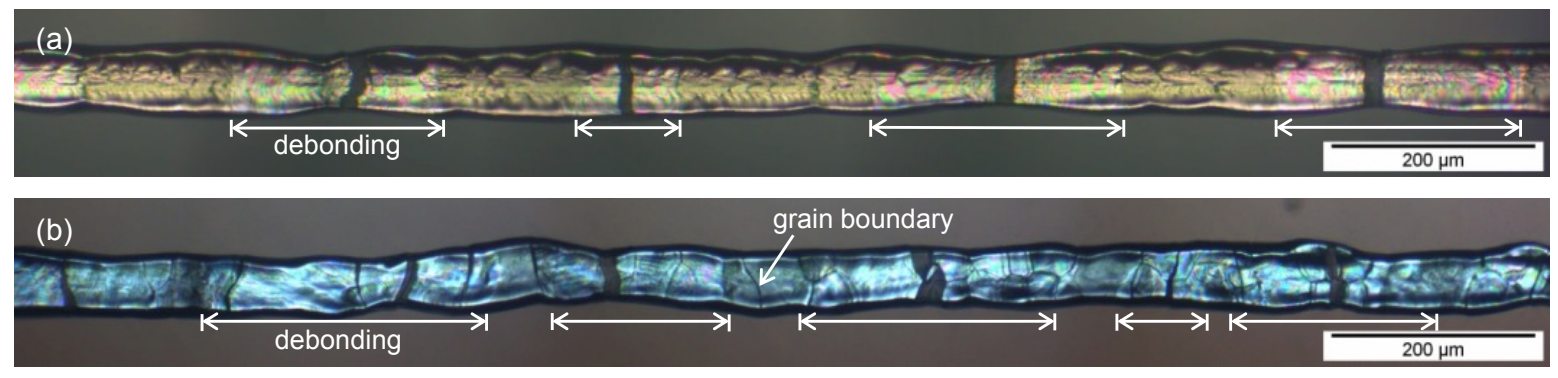

Fig. 7. Micrographs of a fractured as-spun (a) and annealed (b) fibre embedded in an epoxy matrix after the fragmentation test. During subsequent loading after the onset of multiple resin necking, debonding took place around the fractures. The annealed fibre broke at the grain boundaries.

The model to determine the IFSS from single fibre fragmentation tests, used due to its simplicity, considers a force balance between the shear force at the fibre/matrix interface and the axial force in the fibre [18]:

$$
I F S S=\frac{d\langle\sigma\rangle}{2 l_{c}},
$$

where $d$ is the fibre diameter, $\langle\sigma\rangle$ the average fibre strength, and $l_{c}$ the critical stress transfer length of the fibre. Assuming an even distribution of the fragments lengths in the range $l_{c} / 2<l<l_{c}$, the critical fibre length is given by [19]:

$$
l_{c}=\frac{4}{3}\langle l\rangle
$$

where $\langle l\rangle$ is the average fragment length at saturation of the fragmentation process. The fibre strength for the corresponding fragment length was calculated using Eq. (2) and the Weibull parameters determined from single fibre tension tests. Assuming that the 
smallest value of $\langle l\rangle$ in the fragmentation diagram (Fig. 6) corresponds to the saturation level, obtained IFSS values calculated according to Eq. (5) are summarized in Fig. 8.

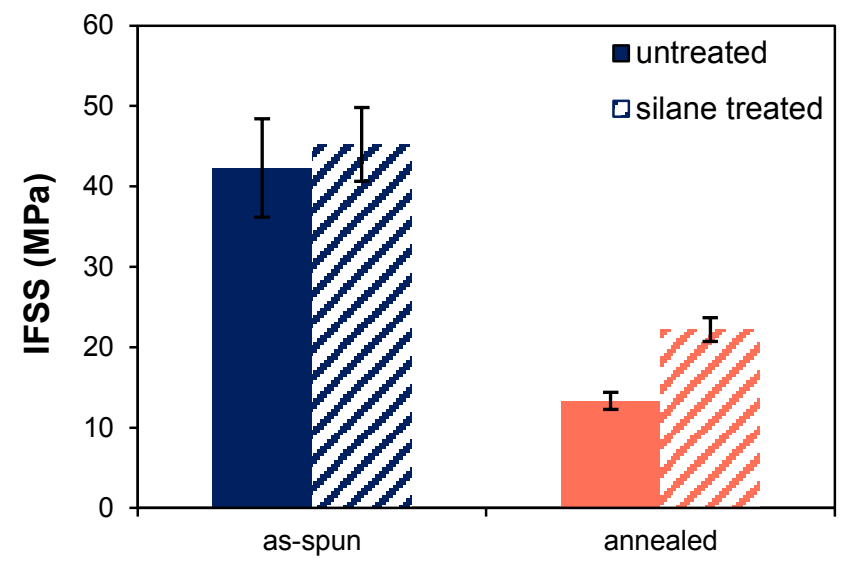

Fig. 8. IFSS values of as-spun and annealed untreated and silane treated Ni-Mn-Ga fibres embedded in epoxy.

For the as-spun, untreated Ni-Mn-Ga fibres embedded in the epoxy matrix, an IFSS of $42.3 \pm 6.1 \mathrm{MPa}$ was found. Annealing considerably reduced the IFSS to $13.3 \pm 1.1 \mathrm{MPa}$, which shows the major influence of the microstructure of the fibre surface. As-spun fibres exhibited a "pimpled" surface caused by the dendritic-like grains, Fig. 1 (a). Annealed fibres, however, had a smooth surface resulting from grain growth and the bamboo structure, Fig. 1 (b). This smoothing of the Ni-Mn-Ga surface during annealing reduced the mechanical interlocking of the surface with the matrix and thus resulted in reduced interfacial shear strength. Such a considerable influence of the mechanical interlocking on the bonding between fibre and epoxy matrix was found as well for thermal shape memory fibres [20]. 
Sylilation did not significantly increase the IFSS in the case of as-spun fibres. In contrast, for the annealed fibres it led to an IFSS increase of $67 \%$.This trend confirms observations of Kauffmann-Weiss et al. [21] on untreated and silane treated Ni-Mn-Ga fibres produced by crucible melt-extraction embedded in an epoxy matrix for pull-out tests. For as-spun fibres, in contrary, a possible effect of the silylation is within the experimental scatter. This could be explained by the fact that the shear yield strength of the resin, responsible for a maximum theoretical value of adhesion, is approached. Indeed, according to the von Mises yield criterion $\tau=\sigma^{*} / \sqrt{3}$ ( $\tau$. shear strength, $\sigma^{*}$ : tensile strength of the resin), the shear strength for the epoxy used in this study (Fig. 6 (a)) is about $40 \mathrm{MPa}$. This corresponds roughly to the IFSS values obtained for as-spun fibres.

Although the obtained IFSS values determined using the constant interfacial shear stress model are reasonable, they may seem erroneous since there was no clear experimental evidence that the saturation of fragmentation was achieved (as it was assumed above). As shown in Fig. 6 (a), even for strains close to the maximum stress of the sample, new fragments still appear. In order to calculate the IFSS in such a case, more advanced models should be applied to verify the accuracy of the constant interfacial shear stress model.

In this work, an analytical model proposed by Andersons et al. [22] that accounts for the effect of finite fibre length on the initial fragmentation as well as for break interaction on the advanced fragmentation stage was used. The input parameters of the model are the radius and Weibull parameters of the fibre as well as fragmentation diagrams. In addition, this model could be used to avoid carrying out time consuming single fibre tension tests, 
since the Weibull parameters can be determined directly from the initial fragmentation stage. The average number of breaks is given by:

$$
\langle N\rangle=\frac{l}{l_{0}}\left[\frac{\sigma(\varepsilon)}{\beta}\right]^{\alpha} .
$$

Since the Ni-Mn-Ga fibres used in this work accumulate quite significant plastic strains during loading, Fig. 3, the secant modulus of the single fibre tension tests was used instead of the Young's modulus to better evaluate the failure stress: $\sigma(\varepsilon)=\left\langle\frac{\sigma}{\varepsilon^{*}}\right\rangle\left(\varepsilon+\varepsilon_{r}\right)$ ( $\varepsilon^{*}$ : stain at failure). Calculations for the fragmentation test data of the as-spun Ni-Mn-Ga fibres lead to the Weibull parameters summarised in Table 1. The shape parameters almost coincide for untreated and silane treated fibres. This remarkable result could be attributed to healing of surface flaws by the resin, akin to the effect of silane treatment described above. However, both shape parameters are smaller than the one of the as-spun, silane treated fibres obtained from the single fibre tension tests. Furthermore, the scale parameter values calculated from the fragmentation test are lower than those determined from the single fibre tension tests. A possible explanation for these reduced values is damage accumulation in the fibre due to the compressive residual stresses in the fragmentation specimens. It is reported for carbon fibres [23] that experimentally determined Weibull parameters are very sensitive to residual stresses caused by a thermal coefficient mismatch in the single fibre composite tests.

Finally, using the determined Weibull parameters, the theoretical model described in Ref. [22] can be applied to fit the experimental data as shown in Fig. 9. (Note that since the 
fibre cross section varies considerably from fibre to fibre, the aspect ratio of the fragments is given on the vertical axis.) The modelling results using the IFSS values shown in Fig. 8 are in good agreement with the experimental data. Thus, the constant interfacial shear stress model, Eq. (5), is adequate to determine the IFSS of the Ni-Mn-Ga fibre/epoxy matrix systems investigated in this study. Furthermore, the analytical curve has achieved the asymptote before the end of fragmentation test confirming that saturation was reached.

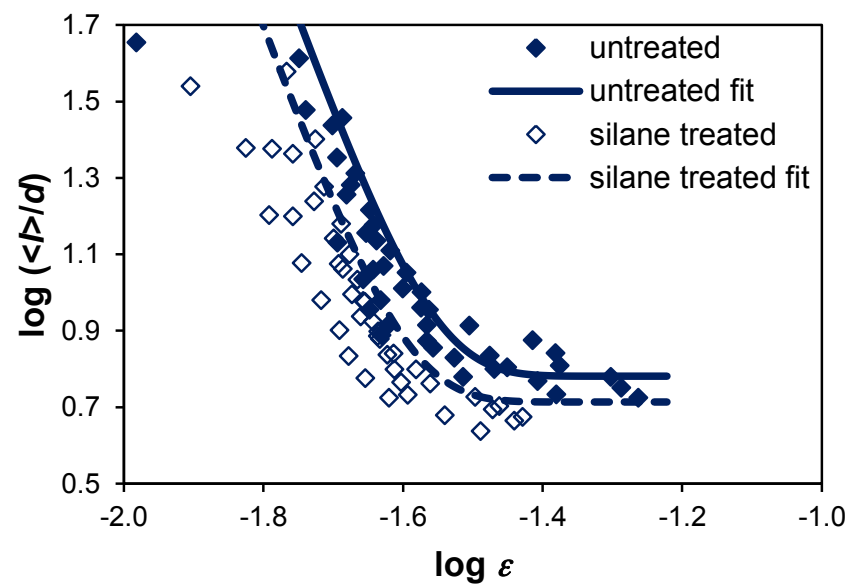

Fig. 9. Normalised average fragment length as function of fibre strain for all as-spun, untreated and silane treated single fibre composites. Solid line: modelling according to Ref. [22].

Regarding the annealed fibres, the analytical model can not be applied due to the lack of fragmentation diagrams. The difference in the calculated IFSS using Eq. (5) however indicates that an advanced stage of fragmentation was achieved, since during the initial fragmentation phase the fragmentation diagrams (as shown in Fig. 6 (b)) should coincide for as-spun and annealed fibres. Therefore it can be concluded that the obtained IFSS values for the annealed fibres are as well quite close to the IFSS value at saturation. 


\section{Conclusions}

Silane treatment of as-spun and annealed melt-spun Ni-Mn-Ga fibres increased their tensile strength and decreased the scatter in strength due to healing of surface flaws and/or forming of a protective surface coating. The twinning in the annealed fibre was, however, not hindered or blocked by the silane coating. For all cases, fracture was brittle and followed a Weibull distribution, although the number of tested samples was low due to availability. As-spun fibres exhibited a rough surface microstructure, resulting in an adhesion with epoxy, $42.3 \pm 6.1 \mathrm{MPa}$ for the untreated and 45.2 $\pm 4.6 \mathrm{MPa}$ for the silane treated fibres, that is in the range of the shear yield strength, about $40 \mathrm{MPa}$, of the resin used in this study. The lower interfacial shear strength of the smooth surfaced bamboostructured annealed fibres was considerably increased, from $13.3 \pm 1.1 \mathrm{MPa}$ to $22.2 \pm 1.5 \mathrm{MPa}$, by the silane treatment. As a result, silane treatment of the bamboostructured Ni-Mn-Ga material is recommended to improve adhesion and stress transfer with epoxy matrices in composite applications.

\section{Acknowledgement}

This work is supported by the Swiss National Science Foundation under the National Research Programme NRP 62 n406240-126120. E. Spārniņš gratefully acknowledges the support of CRUS Sciex Programme NMS-CH, Project Code: 12.217. Furthermore, we thank N. Kucza of Boise State University (USA) for producing the Ni-Mn-Ga ingot and Prof. X. Zhang of Harbin Institute of Technology (China) for preparing the melt-spun fibres. 


\section{References}

[1] Heczko O., Sozinov A., Ullakko K., Giant field-induced reversible strain in magnetic shape memory NiMnGa alloy, IEEE Trans. Magn., 36 (2000) 3266-8.

[2] Straka L., Lanska N., Ullakko K., Sozinov A., Twin microstructure dependent mechanical response in Ni-Mn-Ga single crystals, Appl. Phys. Lett., 96 (2010) 131903(1)(3).

[3] Marioni M.A., O'Handley R.C., Allen S.M., Pulsed magnetic field-induced actuation of Ni-Mn-Ga single crystals, Appl. Phys. Lett., 83 (2003) 3966-8.

[4] Dunand D.C., Müllner P., Size Effects on Magnetic Actuation in Ni-Mn-Ga ShapeMemory Alloys, Adv. Mater., 23 (2011) 216-32.

[5] Drzal L.T., Herrera-Franco P.J., Ho H., Fibre-matrix interface tests, in: Kelly A., Zweben C. (Eds.), Comprehensive composite materials, Pergamon Press, New York, 2000.

[6] Wingfield J., Treatment of composite surfaces for adhesive bonding, International journal of adhesion and adhesives, 13 (1993) 151-6.

[7] Golaz B., Michaud V., Månson J.-A., Adhesion of thermoplastic polyurethane elastomer to galvanized steel, International journal of adhesion and adhesives, 31 (2011) 805-15.

[8] Bouchet J., Pax G., Leterrier Y., Michaud V., Manson J., Formation of aminosilaneoxide interphases, Composite Interfaces, 13 (2006) 573-88.

[9] Qian M.F., Zhang X.X., Witherspoon C., Sun J.F., Müllner P., Superelasticity and shape memory effects in polycrystalline $\mathrm{Ni}-\mathrm{Mn}-\mathrm{Ga}$ microwires, J. Alloy. Compd., 577 Supplement 1 (2013) S296-S9.

[10] Glock S., Zhang X., Kucza N., Müllner P., Michaud V., Structural, physical and damping properties of melt-spun $\mathrm{Ni}-\mathrm{Mn}-\mathrm{Ga}$ wire-epoxy composites, Composites Part A: Applied Science and Manufacturing, 63 (2014) 68-75.

[11] Weibull W., A statistical theory for the strength of materials, Ingenjörsvetenskapsakademiens Handlingar, 151 (1939) 1-45.

[12] Benard A., Bos-Levenbach E., The plotting of observations on probability paper, Statistica Neerlandica, 7 (1953) 163-73.

[13] Zinck P., Pay M., Rezakhanlou R., Gerard J., Mechanical characterisation of glass fibres as an indirect analysis of the effect of surface treatment, J. Mater. Sci., 34 (1999) 2121-33. 
[14] Zinck P., Mäder E., Gerard J., Role of silane coupling agent and polymeric film former for tailoring glass fiber sizings from tensile strength measurements, J. Mater. Sci., 36 (2001) 5245-52.

[15] Yang L., Thomason J., Effect of silane coupling agent on mechanical performance of glass fibre, J. Mater. Sci., 48 (2013) 1947-54.

[16] Buchelnikov V.D., Khovailo V.V., Takagi T., The thermal expansion coefficient and volume magnetostriction of Heusler Ni2MnGa alloys, J. Magn. Magn. Mater., 300 (2006) e459-e61.

[17] Rudajevová A., Analysis of the thermal expansion characteristics of $\mathrm{Ni}_{53.6} \mathrm{Mn}_{27.1} \mathrm{Ga}_{19.3}$ alloy, J. Alloy. Compd., 430 (2007) 153-7.

[18] Kelly A., Tyson W.R., Tensile properties of fibre-reinforced metals- copper/tungsten and copper/molybdenum, J. Mech. Phys. Solids, 13 (1965) 329-50.

[19] Ohsawa T., Nakayama A., Miwa M., Hasegawa A., Temperature dependence of critical fiber length for glass fiber-reinforced thermosetting resins, Journal of Applied Polymer Science, 22 (1978) 3203-12.

[20] Schrooten J., Michaud V., Parthenios J., Psarras G.C., Galiotis C., Gotthardt R., Manson J., Van Humbeeck J., Progress on composites with embedded shape memory alloy wires, Mater. Trans., 43 (2002) 961-73.

[21] Kauffmann-Weiss S., Scheerbaum N., Liu J., Klauss H., Schultz L., Mäder E., Häßler R., Heinrich G., Gutfleisch O., Reversible Magnetic Field Induced Strain in Ni2MnGaPolymer-Composites, Advanced Engineering Materials, 14 (2012) 20-7.

[22] Andersons J., Joffe R., Hojo M., Ochiai S., Fibre fragment distribution in a single-fibre composite tension test, Composites Part B: Engineering, 32 (2001) 323-32.

[23] Hui C.Y., Shia D., Berglund L.A., Estimation of interfacial shear strength: an application of a new statistical theory for single fiber composite test, Composites Science and Technology, 59 (1999) 2037-46. 